\title{
CONGRUENCES FOR ANDREWS' SPT-FUNCTION MODULO 32760 AND EXTENSION OF ATKIN'S HECKE-TYPE PARTITION CONGRUENCES
}

\author{
F. G. GARVAN
}

Dedicated to the memory of A.J. (Alf) van der Poorten, my former teacher

\begin{abstract}
New congruences are found for Andrews' smallest parts partition function $\operatorname{spt}(n)$. The generating function for $\operatorname{spt}(n)$ is related to the holomorphic part $\alpha(24 z)$ of a certain weak Maass form $\mathcal{M}(z)$ of weight $\frac{3}{2}$. We show that a normalized form of the generating function for $\operatorname{spt}(n)$ is an eigenform modulo 72 for the Hecke operators $T\left(\ell^{2}\right)$ for primes $\ell>3$, and an eigenform modulo $p$ for $p=5,7$ or 13 provided that $(\ell, 6 p)=1$. The result for the modulus 3 was observed earlier by the author and considered by Ono and Folsom. Similar congruences for higher powers of $p$ (namely $5^{6}, 7^{4}$ and $13^{2}$ ) occur for the coefficients of the function $\alpha(z)$. Analogous results for the partition function were found by Atkin in 1966. Our results depend on the recent result of Ono that $\mathcal{M}_{\ell}(z / 24)$ is a weakly holomorphic modular form of weight $\frac{3}{2}$ for the full modular group where

$$
\mathcal{M}_{\ell}(z)=\mathcal{M}(z) \mid T\left(\ell^{2}\right)-\left(\frac{3}{\ell}\right)(1+\ell) \mathcal{M}(z) .
$$
\end{abstract}

\section{INTRODUCTION}

Andrews [1] defined the function $\operatorname{spt}(n)$ as the number of smallest parts in the partitions of $n$. He related this function to the second rank moment and proved some surprising congruences mod 5,7 and 13. Rank and crank moments were introduced by A. O. L. Atkin and the author [2]. Bringmann [6] studied analytic, asymptotic and congruence properties of the generating function for the second rank moment as a quasi-weak Maass form. Further congruence properties of Andrews' spt-function were found by the author [10, [11, Folsom and Ono 8 and Ono [12]. In particular, Ono [12] proved that if $\left(\frac{1-24 n}{\ell}\right)=1$ then

$$
\operatorname{spt}\left(\ell^{2} n-\frac{1}{24}\left(\ell^{2}-1\right)\right) \equiv 0 \quad(\bmod \ell)
$$

for any prime $\ell \geq 5$. This amazing result was originally conjectured by the author [i]. Earlier special cases were observed by Tina Garrett [9] and her students. Recently the author [11] has proved the following congruences for powers of 5,7 and 13. For $a, b, c \geq 3$,

$$
\begin{array}{ll}
\operatorname{spt}\left(5^{a} n+\delta_{a}\right)+5 \operatorname{spt}\left(5^{a-2} n+\delta_{a-2}\right) \equiv 0 & \left(\bmod 5^{2 a-3}\right), \\
\operatorname{spt}\left(7^{b} n+\lambda_{b}\right)+7 \operatorname{spt}\left(7^{b-2} n+\lambda_{b-2}\right) \equiv 0 & \left(\bmod 7^{\left\lfloor\frac{1}{2}(3 b-2)\right\rfloor}\right),
\end{array}
$$

Date: September 3, 2018.

2010 Mathematics Subject Classification. Primary 11P83, 11F33, 11F37; Secondary 11P82, 05A15, 05 A17.

Key words and phrases. Andrews's spt-function, weak Maass forms, congruences, partitions, modular forms.

The author was supported in part by NSA Grant H98230-09-1-0051. The first draft of this paper was written October 25, 2010.

(i) The congruence (1.1) was first conjectured by the author in a Colloquium given at the University of Newcastle, Australia on July 17, 2008. 


$$
\operatorname{spt}\left(13^{c} n+\gamma_{c}\right)-13 \operatorname{spt}\left(13^{c-2} n+\gamma_{c-2}\right) \equiv 0 \quad\left(\bmod 13^{c-1}\right),
$$

where $\delta_{a}, \lambda_{b}$ and $\gamma_{c}$ are the least nonnegative residues of the reciprocals of $24 \bmod 5^{a}, 7^{b}$ and $13^{c}$ respectively.

As in [12, 11] we define

$$
\mathbf{a}(n):=12 \operatorname{spt}(n)+(24 n-1) p(n),
$$

for $n \geq 0$, and define

$$
\alpha(z):=\sum_{n \geq 0} \mathbf{a}(n) q^{n-\frac{1}{24}},
$$

where as usual $q=\exp (2 \pi i z)$ and $\Im(z)>0$. We note that $\operatorname{spt}(0)=0$ and $p(0)=1$. Bringmann [6] showed that $\alpha(24 z)$ is the holomorphic part of the weight $\frac{3}{2}$ weak Maass form $\mathcal{M}(z)$ on $\Gamma_{0}(576)$ with Nebentypus $\chi_{12}$ where

$$
\mathcal{M}(z):=\alpha(24 z)-\frac{3 i}{\pi \sqrt{2}} \int_{-\bar{z}}^{i \infty} \frac{\eta(24 \tau) d \tau}{(-i(\tau+z))^{\frac{3}{2}}}
$$

$\eta(z):=q^{\frac{1}{24}} \prod_{n=1}^{\infty}\left(1-q^{n}\right)$ is the Dedekind eta-function, the function $\alpha(z)$ is defined in (1.6), and

$$
\chi_{12}(n)=\left\{\begin{array}{lll}
1 & \text { if } n \equiv \pm 1 \quad(\bmod 12) \\
-1 & \text { if } n \equiv \pm 5 \quad(\bmod 12) \\
0 & \text { otherwise }
\end{array}\right.
$$

Ono [12] showed that for $\ell \geq 5$ prime, the operator

$$
T\left(\ell^{2}\right)-\chi_{12}(\ell) \ell(1+\ell)
$$

annihilates the nonholomorphic part of $\mathcal{M}(z)$, and the function $\mathcal{M}_{\ell}(z / 24)$ is a weakly holomorphic modular form of weight $\frac{3}{2}$ for the full modular group where

$$
\mathcal{M}_{\ell}(z)=\mathcal{M}(z)\left|T\left(\ell^{2}\right)-\chi_{12}(\ell)(1+\ell) \mathcal{M}(z)=\alpha(24 z)\right| T\left(\ell^{2}\right)-\chi_{12}(\ell)(1+\ell) \alpha(24 z) .
$$

In fact he obtained

Theorem 1.1 (Ono [12]). If $\ell \geq 5$ is prime then the function

$$
\mathcal{M}_{\ell}(z / 24) \eta(z)^{\ell^{2}}
$$

is an entire modular form of weight $\frac{1}{2}\left(\ell^{2}+3\right)$ for the full modular group $\Gamma(1)$.

Applying this theorem Ono obtained

$$
\mathcal{M}_{\ell}(z) \equiv 0 \quad(\bmod \ell)
$$

The congruence (1.1) then follows easily.

Folsom and Ono [8] sketched the proof of the following

Theorem 1.2 (Folsom and Ono). If $\ell \geq 5$ is prime then

$$
\operatorname{spt}\left(\ell^{2} n-s_{\ell}\right)+\chi_{12}(\ell)\left(\frac{1-24 n}{\ell}\right) \operatorname{spt}(n)+\ell \operatorname{spt}\left(\frac{n+s_{\ell}}{\ell^{2}}\right) \equiv \chi_{12}(\ell)(1+\ell) \operatorname{spt}(n) \quad(\bmod 3)
$$

where

$$
s_{\ell}=\frac{1}{24}\left(\ell^{2}-1\right) \text {. }
$$

This result was observed earlier by the author. In this paper we prove a much stronger result. 
Theorem 1.3. $\quad$ (i) If $\ell \geq 5$ is prime then

(1.15) $\operatorname{spt}\left(\ell^{2} n-s_{\ell}\right)+\chi_{12}(\ell)\left(\frac{1-24 n}{\ell}\right) \operatorname{spt}(n)+\ell \operatorname{spt}\left(\frac{n+s_{\ell}}{\ell^{2}}\right) \equiv \chi_{12}(\ell)(1+\ell) \operatorname{spt}(n) \quad(\bmod 72)$.

(ii) If $\ell \geq 5$ is prime, $t=5,7$ or 13 and $\ell \neq t$ then

$\operatorname{spt}\left(\ell^{2} n-s_{\ell}\right)+\chi_{12}(\ell)\left(\frac{1-24 n}{\ell}\right) \operatorname{spt}(n)+\ell \operatorname{spt}\left(\frac{n+s_{\ell}}{\ell^{2}}\right) \equiv \chi_{12}(\ell)(1+\ell) \operatorname{spt}(n) \quad(\bmod t)$.

Of course this implies the

Corollary 1.4. If $\ell$ is prime and $\ell \notin\{2,3,5,7,13\}$ then

$$
\operatorname{spt}\left(\ell^{2} n-s_{\ell}\right)+\chi_{12}(\ell)\left(\frac{1-24 n}{\ell}\right) \operatorname{spt}(n)+\ell \operatorname{spt}\left(\frac{n+s_{\ell}}{\ell^{2}}\right) \equiv \chi_{12}(\ell)(1+\ell) \operatorname{spt}(n) \quad(\bmod 32760) .
$$

This congruence modulo $32760=2^{3} \cdot 3^{2} \cdot 5 \cdot 7 \cdot 13$ is the congruence referred in the title of this paper.

In 1966, Atkin [4] found a similar congruence for the partition function.

Theorem 1.5 (Atkin). Let $t=5,7$, or 13 , and $c=6$, 4, or 2 respectively. Suppose $\ell \geq 5$ is prime and $\ell \neq t$. If $\left(\frac{1-24 n}{t}\right)=-1$, then

$$
\ell^{3} p\left(\ell^{2} n-s_{\ell}\right)+\ell \chi_{12}(\ell)\left(\frac{1-24 n}{\ell}\right) p(n)+p\left(\frac{n+s_{\ell}}{\ell^{2}}\right) \equiv \gamma_{t} p(n) \quad\left(\bmod t^{c}\right),
$$

where $\gamma_{t}$ is an integral constant independent of $n$.

We find that there is a corresponding result for the function $\mathbf{a}(n)$ defined in (1.5).

Theorem 1.6. Let $t=5$, 7, or 13 , and $c=6$, 4, or 2 respectively. Suppose $\ell \geq 5$ is prime and $\ell \neq t$. If $\left(\frac{1-24 n}{t}\right)=-1$, then

$$
\mathbf{a}\left(\ell^{2} n-s_{\ell}\right)+\chi_{12}(\ell)\left(\frac{1-24 n}{\ell}\right) \mathbf{a}(n)+\ell \mathbf{a}\left(\frac{n+s_{\ell}}{\ell^{2}}\right) \equiv \chi_{12}(\ell)(1+\ell) \mathbf{a}(n) \quad\left(\bmod t^{c}\right) .
$$

In Section 2 we prove Theorem 1.3 The method involves reviewing the action of weight $-\frac{1}{2}$ Hecke operators $T\left(\ell^{2}\right)$ on the function $\eta(z)^{-1}$ and doing a careful study of the action of weight $\frac{3}{2}$ Hecke operators on the function $\frac{d}{d z} \eta(z)^{-1}$ modulo 5, 7, 13, 27 and 32. In Section 3 we prove Theorem 1.6. The method involves extending Atkin's [4] on modular functions to weight two modular forms on $\Gamma_{0}(t)$ for $t=5,7$ and 13. The proof of both Theorems 1.3 and 1.6 depend on Ono's Theorem 1.1.

\section{Proof of Theorem 1.3}

In this section we prove Theorem 1.3. Atkin [3] showed essentially that applying certain weight $-\frac{1}{2}$ Hecke operators $T\left(\ell^{2}\right)$ to the function $\eta(z)^{-1}$ produces a function with the same multiplier system as $\eta(z)^{-1}$ and thus $\eta(z)$ times this function is a certain polynomial (depending on $\ell$ ) of Klein's modular invariant $j(z)$. We review Ono's [13 recent explicit form for these polynomials. Although our proof does not depend on Ono's result it is quite useful for computational purposes. The action of the corresponding weight $\frac{3}{2}$ Hecke operators on $\frac{d}{d z} \eta(z)^{-1}$ can be given in terms of the same polynomials. See Theorem 2.3 below. To finish the proof of the theorem we need to make a careful study of the action of these operators modulo 5, 7, 13, 27 and 32 .

For $\ell \geq 5$ prime we define

$$
Z_{\ell}(z)=\sum_{n=-s_{\ell}}^{\infty}\left(\ell^{3} p\left(\ell^{2} n-s_{\ell}\right)+\ell \chi_{12}(\ell)\left(\frac{1-24 n}{\ell}\right) p(n)+p\left(\frac{n+s_{\ell}}{\ell^{2}}\right)\right) q^{n-\frac{1}{24}} .
$$


Proposition 2.1 (Atkin [4]). The function $Z_{\ell}(z) \eta(z)$ is a modular function on the full modular group $\Gamma(1)$.

It follows that $Z_{\ell}(z) \eta(z)$ is a polynomial in $j(z)$, where $j(z)$ is Klein's modular invariant

$$
j(z):=\frac{E_{4}(z)^{2}}{\Delta(z)}=q^{-1}+744+196884 q+\cdots,
$$

$E_{2}(z), E_{4}(z), E_{6}(z)$ are the usual Eisenstein series

$$
E_{2}(z):=1-24 \sum_{n=1}^{\infty} \sigma_{1}(n) q^{n}, \quad E_{4}(z):=1+240 \sum_{n=1}^{\infty} \sigma_{3}(n) q^{n}, \quad E_{6}(z):=1-504 \sum_{n=1}^{\infty} \sigma_{5}(n) q^{n},
$$

$\sigma_{k}(n)=\sum_{d \mid n} q^{k}$, and $\Delta(z)$ is Ramanujan's function

$$
\Delta(z):=\eta(z)^{24}=q \prod_{n=1}^{\infty}\left(1-q^{n}\right)^{24} .
$$

In a recent paper, Ono 13 has found a nice formula for this polynomial. We define

$$
E(q):=\prod_{n=1}^{\infty}\left(1-q^{n}\right)=q^{-\frac{1}{24}} \eta(z)
$$

and a sequence of polynomials $A_{m}(x) \in \mathbb{Z}[x]$ by

$$
\begin{aligned}
\sum_{m=0}^{\infty} A_{m}(x) q^{m} & =E(q) \frac{E_{4}(z)^{2} E_{6}(z)}{\Delta(z)} \frac{1}{j(z)-x} \\
& =1+(x-745) q+\left(x^{2}-1489 x+160511\right) q^{2}+\cdots .
\end{aligned}
$$

Theorem 2.2 (Ono [13]). For $\ell \geq 5$ prime

$$
Z_{\ell}(z) \eta(z)=\ell \chi_{12}(\ell)+A_{s_{\ell}}(j(z)),
$$

where $Z_{\ell}(z)$ is given in (2.1), and $s_{\ell}$ is given in (1.14).

We define a sequence of polynomials $C_{\ell}(x) \in \mathbb{Z}[x]$ by

$$
\begin{aligned}
C_{\ell}(x) & :=\ell \chi_{12}(\ell)+A_{s_{\ell}}(x), \\
& =\sum_{n=0}^{s_{\ell}} c_{n, \ell} x^{n},
\end{aligned}
$$

so that

$$
Z_{\ell}(z) \eta(z)=C_{\ell}(j(z))
$$

We define

$$
d(n):=(24 n-1) p(n),
$$

so that

$$
\sum_{n=0}^{\infty} d(n) q^{24 n-1}=q \frac{d}{d q} \frac{1}{\eta(24 z)}=-\frac{E_{2}(24 z)}{\eta(24 z)},
$$

and

$$
\mathbf{a}(n)=12 \operatorname{spt}(n)+d(n) .
$$


For $\ell \geq 5$ prime we define

$$
\Xi_{\ell}(z)=\sum_{n=-s_{\ell}}^{\infty}\left(d\left(\ell^{2} n-s_{\ell}\right)+\chi_{12}(\ell)\left(\left(\frac{1-24 n}{\ell}\right)-1-\ell\right) d(n)+\ell d\left(\frac{n+s_{\ell}}{\ell^{2}}\right)\right) q^{n-\frac{1}{24}}
$$

We then have the following analogue of Theorem 2.2

Theorem 2.3. For $\ell \geq 5$ prime we have

$$
\begin{aligned}
\ell \Xi_{\ell}(z) \eta(z) \Delta(z)^{s_{\ell}}=- & \sum_{n=0}^{s_{\ell}} c_{n, \ell} E_{4}(z)^{3 n-1} \Delta(z)^{s_{\ell}-n}\left(24 n E_{6}(z)+E_{4}(z) E_{2}(z)\right) \\
& +\chi_{12}(\ell) \ell(1+\ell) E_{2}(z) \Delta(z)^{s_{\ell}}
\end{aligned}
$$

where the coefficients $c_{n, \ell}$ are defined by (2.6) and (2.8).

Proof. Suppose $\ell \geq 5$ is prime. In equation (2.9) we replace $z$ by $24 z$, apply the operator $q \frac{d}{d q}$ and replace $z$ by $\frac{1}{24} z$ to obtain

$$
\ell \Xi_{\ell}(z) \eta(z)=24 C_{\ell}^{\prime}(j(z)) q \frac{d}{d q}(j(z))+\left(\chi_{12}(\ell) \ell(1+\ell)-C_{\ell}(j(z)) E_{2}(z)\right.
$$

The result then follows easily from the identities

$$
j(z) \Delta(z)=E_{4}(z)^{3}, \quad q \frac{d}{d q}(\Delta(z))=\Delta(z) E_{2}(z), \quad \text { and } \quad q \frac{d}{d q}(j(z)) \Delta(z)=-E_{4}(z)^{2} E_{6}(z),
$$

which we leave as an easy exercise.

We are now ready to prove Theorem 1.3. A standard calculation gives the following congruences.

$$
E_{4}(z)^{3}-720 \Delta(z) \equiv 1 \quad(\bmod 65520), \quad \text { and } \quad E_{2}(z) \equiv E_{4}(z)^{2} E_{6}(z) \quad(\bmod 65520) .
$$

We now use (2.17) to reduce (2.15) modulo 65520 .

$$
\begin{aligned}
& \ell \Xi_{\ell}(z) \eta(z) \Delta(z)^{s_{\ell}} \\
& \equiv-\sum_{n=0}^{s_{\ell}} c_{n, \ell} E_{4}(z)^{3 n-1} \Delta(z)^{s_{\ell}-n}\left(24 n E_{6}(z)\left(E_{4}(z)^{3}-720 \Delta(z)\right)+E_{4}(z)^{3} E_{6}(z)\right) \\
& +\chi_{12}(\ell) \ell(1+\ell) E_{4}(z)^{2} E_{6}(z) \Delta(z)^{s_{\ell}} \quad(\bmod 65520) \\
& \equiv-\sum_{n=0}^{s_{\ell}}(24 n+1) c_{n, \ell} E_{4}(z)^{3 n+2} E_{6}(z) \Delta(z)^{s_{\ell}-n} \\
& +\sum_{n=0}^{s_{\ell}} 720 \cdot 24 n c_{n, \ell} E_{4}(z)^{3 n-1} E_{6}(z) \Delta(z)^{s_{\ell}-n+1}+\chi_{12}(\ell) \ell(1+\ell) E_{4}(z)^{2} E_{6}(z) \Delta(z)^{s_{\ell}} \quad(\bmod 65520) \\
& \equiv\left(720 c_{1, \ell}-c_{0, \ell}+\chi_{12}(\ell) \ell(1+\ell)\right) E_{4}(z)^{2} E_{6}(z) \Delta(z)^{s \ell} \\
& +\sum_{n=1}^{s_{\ell}-1}\left(720 \cdot 24(n+1) c_{n+1, \ell}-(24 n+1) c_{n, \ell}\right) E_{4}(z)^{3 n+2} E_{6}(z) \Delta(z)^{s_{\ell}-n} \\
& -\left(24 s_{\ell}+1\right) c_{s_{\ell}} E_{4}(z)^{3 s_{\ell}+2} E_{6}(z) \quad(\bmod 65520) \text {. }
\end{aligned}
$$


We define

$$
\mathcal{A}_{\ell}(z):=\sum_{n=-s_{\ell}}^{\infty}\left(\mathbf{a}\left(\ell^{2} n-s_{\ell}\right)+\chi_{12}(\ell)\left(\left(\frac{1-24 n}{\ell}\right)-1-\ell\right) \mathbf{a}(n)+\ell \mathbf{a}\left(\frac{n+s_{\ell}}{\ell^{2}}\right)\right) q^{n-\frac{1}{24}}
$$

and

$$
\mathcal{S}_{\ell}(z):=\sum_{n=1}^{\infty}\left(\operatorname{spt}\left(\ell^{2} n-s_{\ell}\right)+\chi_{12}(\ell)\left(\left(\frac{1-24 n}{\ell}\right)-1-\ell\right) \operatorname{spt}(n)+\ell \operatorname{spt}\left(\frac{n+s_{\ell}}{\ell^{2}}\right)\right) q^{n-\frac{1}{24}},
$$

so that

$$
\mathcal{A}_{\ell}(z)=12 \mathcal{S}_{\ell}(z)+\Xi_{\ell}(z)=\mathcal{M}_{\ell}(z / 24) .
$$

By Theorem 1.1 and equation (1.10) we see that the function

$$
\ell \mathcal{A}_{\ell}(z) \eta(z) \Delta(z)^{s \ell} \in M_{\frac{1}{2}\left(\ell^{2}+3\right)}(\Gamma(1)),
$$

the space of entire modular forms of weight $\frac{1}{2}\left(\ell^{2}+3\right)$ on $\Gamma(1)$. Since $\frac{1}{2}\left(\ell^{2}+3\right)=2+12 s_{\ell}$ the set

$$
\left\{E_{4}(z)^{3 n-1} E_{6}(z) \Delta(z)^{s_{\ell}-n}: 1 \leq n \leq s_{\ell}\right\}
$$

is a basis. Hence there are integers $b_{n, \ell}\left(1 \leq n \leq s_{\ell}\right)$ such that

$$
\mathcal{A}_{\ell}(z) \eta(z) \Delta(z)^{s_{\ell}}=\sum_{n=1}^{s_{\ell}} b_{n, \ell} E_{4}(z)^{3 n-1} E_{6}(z) \Delta(z)^{s_{\ell}-n} .
$$

Using (2.17) we find that

$$
\begin{aligned}
\mathcal{A}_{\ell}(z) \eta(z) \Delta(z)^{s_{\ell}} \equiv- & 720 b_{1, \ell} E_{4}(z)^{2} E_{6}(z) \Delta(z)^{s_{\ell}} \\
& +\sum_{n=1}^{s_{\ell}-1}\left(b_{n, \ell}-720 b_{n+1, \ell}\right) E_{4}(z)^{3 n+2} E_{6}(z) \Delta(z)^{s_{\ell}-n} \\
& +b_{s_{\ell}, \ell} E_{4}(z)^{3 s_{\ell}+2} E_{6}(z) \quad(\bmod 65520) .
\end{aligned}
$$

By (2.18), (2.21) and (2.24) we deduce that there are integers $a_{n, \ell}\left(0 \leq n \leq s_{\ell}\right)$ such that

$$
12 \ell \mathcal{S}_{\ell}(z) \eta(z) \Delta(z)^{s_{\ell}} \equiv \sum_{n=0}^{s_{\ell}} a_{n, \ell} E_{4}(z)^{3 n+2} E_{6}(z) \Delta(z)^{s_{\ell}-n} \quad(\bmod 65520) .
$$

It follows that

$$
12 \ell \mathcal{S}_{\ell}(z) \equiv 0 \quad(\bmod 65520)
$$

since

$$
\begin{aligned}
\operatorname{ord}_{i \infty}\left(12 \ell \mathcal{S}_{\ell}(z) \eta(z) \Delta(z)^{s_{\ell}}\right) & =s_{\ell}+1, \\
0 \leq \operatorname{ord}_{i \infty}\left(E_{4}(z)^{3 n+2} E_{6}(z) \Delta(z)^{s_{\ell}-n}\right) & \leq s_{\ell}, \\
E_{4}(z)^{3 n+2} E_{6}(z) \Delta(z)^{s_{\ell}-n} & =q^{s_{\ell}-n}+\cdots,
\end{aligned}
$$

for $0 \leq n \leq s_{\ell}$ and all functions have integral coefficients. Since $65550=2^{4} \cdot 3^{2} \cdot 5 \cdot 7 \cdot 13$, the congruence (2.27) implies Part (ii) of Theorem 1.3. To prove Part (i) we need to work a little harder. We note that the congruence (2.27) does imply

$$
\mathcal{S}_{\ell}(z) \equiv 0 \quad(\bmod 12) \text {. }
$$

We need to show this congruence actually holds modulo 72 . 
First we show the congruence holds modulo 8 by studying $\Xi_{\ell}(z)$ modulo 32 . We need the congruences,

$$
E_{2}(z) \equiv E_{4}(z) E_{6}(z)+16 \Delta(z) \quad(\bmod 32), \quad \text { and } \quad E_{4}(z)^{2} \equiv 1 \quad(\bmod 32),
$$

which are routine to prove. We proceed as in the proof of (2.18) to find that

$$
\begin{aligned}
& \ell \Xi_{\ell}(z) \eta(z) \Delta(z)^{s_{\ell}} \\
& \equiv\left(\chi_{12}(\ell) \ell(1+\ell)-c_{0, \ell}-16 c_{1, \ell}\right) E_{2}(z) \Delta(z)^{s_{\ell}} \\
& \quad-\sum_{n=1}^{s_{\ell}-1}\left((24 n+1) c_{n, \ell}+16 c_{n+1, \ell}\right) E_{4}(z)^{3 n-1} E_{6}(z) \Delta(z)^{s_{\ell}-n} \\
& \quad-\left(24 s_{\ell}+1\right) c_{s_{\ell}} E_{4}(z)^{3 s_{\ell}-1} E_{6}(z) \quad(\bmod 32) .
\end{aligned}
$$

By (2.31), (2.21) and (2.24) we deduce that there are integers $a_{n, \ell}^{\prime}\left(0 \leq n \leq s_{\ell}\right)$ such that

$$
12 \ell \mathcal{S}_{\ell}(z) \eta(z) \Delta(z)^{s_{\ell}} \equiv \sum_{n=1}^{s_{\ell}} a_{n, \ell}^{\prime} E_{4}(z)^{3 n-1} E_{6}(z) \Delta(z)^{s_{\ell}-n}+a_{0, \ell}^{\prime} E_{2}(z) \Delta(z)^{s_{\ell}} \quad(\bmod 32) .
$$

Arguing as before, it follows that

$$
12 \mathcal{S}_{\ell}(z) \equiv 0 \quad(\bmod 32), \quad \text { and } \quad \mathcal{S}_{\ell}(z) \equiv 0 \quad(\bmod 8) .
$$

To complete the proof, we need to study $\Xi_{\ell}(z)$ modulo 27 . We need the congruences,

$$
E_{2}(z) \equiv E_{4}(z)^{5}+18 \Delta(z) \quad(\bmod 27) \quad \text { and } \quad E_{6}(z) \equiv E_{4}(z)^{6} \quad(\bmod 27)
$$

which are routine to prove. We proceed as in the proof of (2.18) and (2.31) to find that

$$
\begin{aligned}
& \ell \Xi_{\ell}(z) \eta(z) \Delta(z)^{s_{\ell}} \\
& \equiv\left(\chi_{12}(\ell) \ell(1+\ell)-c_{0, \ell}-18 c_{1, \ell}\right) E_{2}(z) \Delta(z)^{s_{\ell}} \\
& \quad-\sum_{n=1}^{s_{\ell}-1}\left((24 n+1) c_{n, \ell}+18 c_{n+1, \ell}\right) E_{4}(z)^{3 n-1} E_{6}(z) \Delta(z)^{s_{\ell}-n} \\
& \quad-\left(24 s_{\ell}+1\right) c_{s_{\ell}} E_{4}(z)^{3 s_{\ell}-1} E_{6}(z) \quad(\bmod 27) .
\end{aligned}
$$

By (2.35), (2.21) and (2.24) we deduce that there are integers $a_{n, \ell}^{\prime \prime}\left(0 \leq n \leq s_{\ell}\right)$ such that

$$
12 \ell \mathcal{S}_{\ell}(z) \eta(z) \Delta(z)^{s_{\ell}} \equiv \sum_{n=1}^{s_{\ell}} a_{n, \ell}^{\prime \prime} E_{4}(z)^{3 n-1} E_{6}(z) \Delta(z)^{s_{\ell}-n}+a_{0, \ell}^{\prime \prime} E_{2}(z) \Delta(z)^{s_{\ell}} \quad(\bmod 27) .
$$

Arguing as before, it follows that

$$
12 \mathcal{S}_{\ell}(z) \equiv 0 \quad(\bmod 27), \quad \text { and } \quad \mathcal{S}_{\ell}(z) \equiv 0 \quad(\bmod 9) .
$$

The congruences (2.33) and (2.37) give (1.15) and this completes the proof of Theorem 1.3 .

\section{Proof of Theorem 1.6}

In this section we prove Theorem 1.6. Atkin [4] proved Theorem 1.5 by constructing certain special modular functions on $\Gamma_{0}(t)$ and $\Gamma_{0}\left(t^{2}\right)$ for $t=5,7$ and 13 . We attack the problem by extending Atkin's results to the corresponding weight 2 case.

Let $G L_{2}^{+}(\mathbb{R})$ denote the group of all real $2 \times 2$ matrices with positive determinant. $G L_{2}^{+}(\mathbb{R})$ acts on the complex upper half plane $\mathcal{H}$ by linear fractional transformations. We define the slash 
operator for modular forms of integer weight. Let $k \in \mathbb{Z}$. For a function $f: \mathcal{H} \longrightarrow \mathbb{C}$ and $L=\left(\begin{array}{ll}a & b \\ c & d\end{array}\right) \in G L_{2}^{+}(\mathbb{R})$ we define

$$
\left.f(z)\right|_{k} L=\left.f\right|_{k} L=f \mid L=(\operatorname{det} L)^{\frac{k}{2}}(c z+d)^{-k} f(L z) .
$$

Let $\Gamma^{\prime} \subset \Gamma(1)$ (a subgroup of finite index). We say $f(z)$ is a weakly holomorphic modular form of weight $k$ on $\Gamma^{\prime}$ if $f(z)$ is holomorphic on the upper half plane $\mathcal{H},\left.f(z)\right|_{k} L=f(z)$ for all $L$ in $\Gamma^{\prime}$, and $f(z)$ has at most polar singularities in the local variables at the cusps of the fundamental region of $\Gamma^{\prime}$. We say $f(z)$ is a weakly holomorphic modular function if it is a weakly holomorphic modular form of weight 0 . We say $f(z)$ is an entire modular form of weight $k$ on $\Gamma^{\prime}$ if it is a weakly holomorphic modular form that is holomorphic at the cusps of the fundamental region of $\Gamma^{\prime}$. We denote the space of entire modular forms of weight $k$ on $\Gamma^{\prime}$ by $M_{k}\left(\Gamma^{\prime}\right)$.

Suppose that $t \geq 5$ is prime. We need

$$
\begin{gathered}
W_{t}=W=\left(\begin{array}{cc}
0 & -1 \\
t & 0
\end{array}\right), \quad R=\left(\begin{array}{cc}
1 & 0 \\
-1 & 1
\end{array}\right), \quad V_{a}=\left(\begin{array}{cc}
a & \lambda \\
t & a^{\prime}
\end{array}\right), \quad B_{t}=\left(\begin{array}{ll}
t & 0 \\
0 & 1
\end{array}\right), \\
T_{b, t}=\left(\begin{array}{cc}
1 & b \\
0 & 1
\end{array}\right), \quad Q_{b, t}=\left(\begin{array}{cc}
1 / t & b / t \\
0 & 1
\end{array}\right),
\end{gathered}
$$

where for $1 \leq a \leq t-1, a^{\prime}$ is uniquely defined by $1 \leq a^{\prime} \leq t-1$, and $a^{\prime} a-\lambda t=1$. We have

$$
\begin{aligned}
B_{t} R^{a t} & =W_{t} V_{a} T_{-a^{\prime} / t} \\
R^{a t} W_{t} & =W_{t^{2}} Q_{a, t} .
\end{aligned}
$$

We define

$$
\Phi_{t}(z)=\Phi(z):=\frac{\eta(z)}{\eta\left(t^{2} z\right)} .
$$

Then $\Phi_{t}(z)$ is a modular function of $\Gamma_{0}(t)$,

$$
\Phi_{t}(z) \mid W_{t^{2}}=t \Phi_{t}(z)^{-1} \quad([4,(24)]),
$$

and

$$
\Phi_{t}(z) \mid R^{a t}=\sqrt{t} e^{\pi i(t-1) / 4} e^{-\pi i a^{\prime} t / 12}\left(\frac{a^{\prime}}{t}\right) \frac{\eta(z)}{\eta\left(z-a^{\prime} / t\right)} \quad \text { ([4, (25)]). }
$$

Although $E_{2}(z)$ is not a modular form, it well-known that

$$
\mathcal{E}_{2, t}(z):=\frac{1}{t-1}\left(t E_{2}(t z)-E_{2}(z)\right),
$$

is an entire modular form of weight 2 on $\Gamma_{0}(t)$ and

$$
\mathcal{E}_{2, t}(z) \mid W_{t}=-\mathcal{E}_{2, t}(z) .
$$

Proposition 3.1. Suppose $t \geq 5$ is prime, $K(z)$ is a weakly holomorphic modular function on $\Gamma_{0}(t)$, and

$$
S(z)=\mathcal{E}_{2, t}(t z) K^{*}(t z) \frac{\eta(z)}{\eta\left(t^{2} z\right)}-\chi_{12}(t) \eta(z) \sum_{n=m}^{\infty}\left(\frac{1-24 n}{t}\right) \beta_{t}(n) q^{n-\frac{1}{24}}
$$

where

$$
\mathcal{E}_{2, t}(z) \frac{K(z)}{\eta(z)}=\sum_{n=m}^{\infty} \beta_{t}(n) q^{n-\frac{1}{24}}
$$


and

$$
K^{*}(z)=K(z) \mid W_{t} .
$$

Then $S(z)$ is a weakly holomorphic modular form of weight 2 on $\Gamma_{0}(t)$.

Proof. Suppose $t \geq 5$ is prime and $K(z), K^{*}(z), S(z)$ are defined as in the statement of the proposition. The function

$$
H(z):=\mathcal{E}_{2, t}(t z) \Phi_{t}(z) K^{*}(t z)
$$

is a weakly holomorphic modular form of weight 2 on $\Gamma_{0}\left(t^{2}\right)$. As in [4, Lemma1] the function

$$
S_{1}(z):=\sum_{a=0}^{t-1} H(z) \mid R^{a t}
$$

is a weakly holomorphic modular form of weight 2 on $\Gamma_{0}(t)$. Utilizing (3.2), (3.6), (3.8) and the evaluation of a quadratic Gauss sum [5, (1.7)] we find that

$$
S_{1}(z)=\mathcal{E}_{2, t}(t z) K^{*}(t z) \frac{\eta(z)}{\eta\left(t^{2} z\right)}-\frac{1}{\sqrt{t}} e^{\pi i(t-1) / 4} \eta(z) \sum_{a^{\prime}=1}^{t-1} e^{-\pi i a^{\prime} t / 12}\left(\frac{a^{\prime}}{t}\right) \mathcal{E}_{2, t}\left(z-a^{\prime} / t\right) \frac{K\left(z-a^{\prime} / t\right)}{\eta\left(z-a^{\prime} / t\right)}
$$

Here we have also used the fact that

$$
\mathcal{E}_{2, t}(z) \mid R^{a t}=-\frac{1}{t} \mathcal{E}_{2, t}\left(z-a^{\prime} / t\right),
$$

where $a a^{\prime} \equiv 1(\bmod t)$. Hence

$$
S_{1}(z)=S(z)
$$

This gives the result.

We illustrate Proposition 3.1 with two examples:

$$
S(z)=\mathcal{E}_{2,5}(z)\left(\frac{\eta(z)}{\eta(5 z)}\right)^{6} \quad(K(z)=1 \text { and } t=5)
$$

and

$$
S(z)=\mathcal{E}_{2,7}(z)\left(\left(\frac{\eta(z)}{\eta(7 z)}\right)^{8}+3\left(\frac{\eta(z)}{\eta(7 z)}\right)^{4}\right) \quad(K(z)=1 \text { and } t=7) .
$$

Corollary 3.2. Suppose $t \geq 5$ is prime and $S(z), K(z)$ and the sequence $\beta_{t}(n)$ are defined as in Proposition 3.1. Then

$$
S(z) \mid W_{t}=-\eta(t z) \sum_{t n-s_{t} \geq m} \beta_{t}\left(t n-s_{t}\right) q^{n-\frac{t}{24}} .
$$

Proof. The result follows easily from (3.3), (3.5) and (3.8).

We illustrate the corollary by applying $W$ to both sides of the equations (3.16)-(3.17):

$$
\sum_{\substack{n=1 \\ \text { and }}}^{\infty} \beta_{5}(5 n-1) q^{n-\frac{5}{24}}=5^{3} \frac{\mathcal{E}_{2,5}(z)}{\eta(5 z)}\left(\frac{\eta(5 z)}{\eta(z)}\right)^{6} \quad(K(z)=1 \text { and } t=5)
$$




$$
\sum_{n=1}^{\infty} \beta_{7}(7 n-2) q^{n-\frac{7}{24}}=7^{2} \frac{\mathcal{E}_{2,7}(z)}{\eta(7 z)}\left(3\left(\frac{\eta(7 z)}{\eta(z)}\right)^{4}+7^{2}\left(\frac{\eta(7 z)}{\eta(z)}\right)^{8}\right) \quad(K(z)=1 \text { and } t=7) .
$$

For $t$ and $K(z)$ as in Proposition 3.1 we define

$$
\begin{gathered}
\Psi_{t, K}(z)=\Psi_{t}(z)=\mathcal{E}_{2, t}(t z) \frac{K^{*}(t z)}{\eta\left(t^{2} z\right)}-\chi_{12}(t) \sum_{n=m}^{\infty}\left(\frac{1-24 m}{t}\right) \beta_{t}(n) q^{n-\frac{1}{24}} \\
-\sum_{t^{2} n-s_{t} \geq m} \beta_{t}\left(t^{2} n-s_{t}\right) q^{n-\frac{1}{24}}
\end{gathered}
$$

where $K^{*}(z)$ and the sequence $\beta_{t}(n)$ is defined in (3.10)-(3.11). We have the following analogue of 2.1 .

Corollary 3.3. The function $\Psi_{t, K}(z) \eta(z)$ is a weakly holomorphic modular form of weight 2 on the full modular group $\Gamma(1)$.

Proof. Let $S(z)$ be defined as in (3.9), so that $S(z)$ is a weakly holomorphic modular form of weight 2 on $\Gamma_{0}(t)$. By [14, Lemma 7], the function

$$
S(z)+S(z)\left|W_{t}\right| U
$$

is a modular form of weight 2 on $\Gamma(1)$. Here $U=U_{t}$ is the Atkin operator

$$
g(z) \mid U_{t}=\frac{1}{t} \sum_{a=0}^{t-1} g\left(\frac{z+a}{t}\right) .
$$

The result then follows from applying the $U$-operator to equation (3.18).

We illustrate the $K(z)=1$ case of Corollary 3.3 with two examples:

$$
\Psi_{5}(z)=\frac{E_{4}(z)^{2} E_{6}(z)}{\eta(z)^{25}}
$$

and

$$
\Psi_{7}(z)=\frac{1}{\eta(z)^{49}}\left(E_{4}(z)^{5} E_{6}(z)-745 E_{4}(z)^{2} E_{6}(z) \Delta(z)\right) .
$$

We need a weight 2 analogue of [4, Lemma 3]. For $t=5,7$ or 13 the genus of $\Gamma_{0}(t)$ is zero, and a Hauptmodul is

$$
G_{t}(z):=\left(\frac{\eta(z)}{\eta(t z)}\right)^{24 /(t-1)} .
$$

This function satisfies

$$
G_{t}\left(\frac{-1}{t z}\right)=t^{12 /(t-1)} G_{t}(z)^{-1} .
$$

Proposition 3.4. Suppose $t=5,7$ or 13 , and let $m$ be any negative integer such that $24 m \not \equiv 1$ $(\bmod t)$. Suppose constants $k_{j}(1 \leq j \leq-m)$ are chosen so that

$$
\beta_{t}(n)=0, \quad \text { for } m+1 \leq n \leq-1,
$$

where

$$
K(z)=G_{t}(z)^{-m}+\sum_{k=1}^{-m-1} k_{j} G_{t}(z)^{j}
$$


and

$$
\sum_{n=m}^{\infty} \beta_{t}(n) q^{n-\frac{1}{24}}=\mathcal{E}_{2, t}(z) \frac{K(z)}{\eta(z)} .
$$

Then

$$
\beta_{t}(n)=0, \quad \text { for } \quad\left(\frac{1-24 n}{t}\right)=-\left(\frac{1-24 m}{t}\right) .
$$

Proof. Suppose $t=5,7$ or 13 , and $m$ is a negative integer such that $24 m \not \equiv 1(\bmod t)$. Suppose $K(z)$ is chosen so that (3.28) holds. Let $S(z)$ be defined as in (3.9), and define

$$
B(z):=S(z)+\chi_{12}(t)\left(\frac{1-24 m}{t}\right) \mathcal{E}_{2, t}(z) K(z)
$$

so that

$$
B(z) \mid W_{t}=S^{*}(z)-\chi_{12}(t)\left(\frac{1-24 m}{t}\right) \mathcal{E}_{2, t}(z) K^{*}(z),
$$

where

$$
S^{*}(z)=S(z) \mid W_{t} .
$$

Since $24 m \not \equiv 1(\bmod t)$, we see that

$$
\operatorname{ord}_{0}(S(z))=\operatorname{ord}_{i \infty}\left(S^{*}(z)\right)>0 .
$$

From (3.27) and (3.29) we see that

$$
\operatorname{ord}_{0}(K(z))=\operatorname{ord}_{i \infty}\left(K^{*}(z)\right)>0
$$

and hence

$$
\operatorname{ord}_{0}(B(z))>0 \text {. }
$$

Now

$$
\operatorname{ord}_{i \infty}\left(\mathcal{E}_{2, t}(t z) K^{*}(t z) \frac{\eta(z)}{\eta\left(t^{2} z\right)}\right) \geq t-\frac{1}{24}\left(t^{2}-1\right)>0,
$$

for $t=5,7,13$. By construction the coefficient of $q^{m}$ in $B(z)$ is zero and so (3.28), (3.38) imply that

$$
\operatorname{ord}_{i \infty}(B(z)) \geq 0 \text {. }
$$

Therefore $B(z)$ is an entire modular form of weight 2 and hence a multiple of $\mathcal{E}_{2, t}(z)$ since there are no nontrivial cusp forms of weight 2 on $\Gamma_{0}(t)$ for $t=5,7$ or 13 by [7]. This implies that $B(z)$ is identically zero by (3.37). Hence

$$
\frac{B(z)}{E(q)}=q^{-s_{t}} \mathcal{E}_{2, t}(t z) K^{*}(t z) \frac{1}{E\left(q^{t^{2}}\right)}-\chi_{12}(t) \sum_{n=m}^{\infty}\left(\left(\frac{1-24 n}{t}\right)-\left(\frac{1-24 m}{t}\right)\right) \beta_{t}(n) q^{n}=0
$$

Since $-24 s_{t}-1 \equiv 0(\bmod t)$, this implies that $\beta_{t}(n)=0$ whenever $\left(\frac{1-24 n}{t}\right)=-\left(\frac{1-24 m}{t}\right)$.

We illustrate Proposition 3.4 with two examples:

$$
\begin{aligned}
& \sum_{n=-2}^{\infty} \beta_{5}(n) q^{n}=\frac{\mathcal{E}_{2,5}(z)}{E(q)}\left(G_{5}(z)^{2}+5 G_{5}(z)\right) \\
& =q^{-2}+1-379 q^{3}+625 q^{4}+869 q^{5}-20125 q^{8}+23125 q^{9}+25636 q^{10}-329236 q^{13}+\cdots
\end{aligned}
$$


F. G. GARVAN

In this example, $t=5$ and $m=-2$, and we see that $\beta_{5}(n)=0$ for $n \equiv 1,2(\bmod 5)$. In our second example, $t=7$ and $m=-1$.

$(3.42)$

$$
\sum_{n=-1}^{\infty} \beta_{7}(n) q^{n}=\frac{\mathcal{E}_{2,7}(z)}{E(q)} G_{7}(z)
$$

$$
=q^{-1}+1-15 q^{2}+49 q^{5}-24 q^{6}+88 q^{7}-311 q^{9}+392 q^{12}-182 q^{13}+811 q^{14}-1886 q^{16}+\cdots .
$$

In this example we see that $\beta_{7}(n)=0$ for $n \equiv 1,3,4(\bmod 7)$.

The function

$$
\begin{aligned}
& \frac{E_{4}(z)^{2} E_{6}(z)}{\Delta(z)}=\frac{E_{6}(z)}{E_{4}(z)} j(z) \\
& \quad=q^{-1}-196884 q-42987520 q^{2}-2592899910 q^{3}-80983425024 q^{4}-1666013203000 q^{5}+\cdots
\end{aligned}
$$

is a modular form of weight 2 on $\Gamma(1)$. As a modular form on $\Gamma_{0}(t)$ it has a simple pole at $i \infty$ and a pole of order $t$ at $z=0$. When $t=5,7$ or 13 , it is straightforward to show that there are integers $a_{j, t}(-1 \leq j \leq t)$ such that

$$
\frac{E_{6}(z)}{E_{4}(z)} j(z)=\mathcal{E}_{2, t}(z) \sum_{j=-1}^{t} a_{j, t} G_{t}(z)^{j} .
$$

For example,

$$
\begin{gathered}
\frac{E_{6}(z)}{E_{4}(z)} j(z)=\mathcal{E}_{2,5}(z)\left(G_{5}(z)-3^{2} \cdot 5^{5} \cdot 7 G_{5}(z)^{-1}-\cdot 2^{3} \cdot 5^{8} \cdot 13 G_{5}(z)^{-2}-\cdot 3^{3} \cdot 5^{10} \cdot 7 G_{5}(z)^{-3}\right. \\
\left.-3 \cdot 2^{3} \cdot 5^{13} G_{5}(z)^{-4}-5^{16} G_{5}(z)^{-5}\right) .
\end{gathered}
$$

Reducing (3.45) $\bmod t^{c}$ we obtain a weight 2 analogue of [4, Lemma 4].

Lemma 3.5. We have

$$
\begin{aligned}
& \frac{E_{6}(z)}{E_{4}(z)} j(z) \equiv \mathcal{E}_{2,5}(z)\left(G_{5}(z)+2 \cdot 31 \cdot 5^{5} G_{5}(z)^{-1}\right) \quad\left(\bmod 5^{8}\right) \\
& \frac{E_{6}(z)}{E_{4}(z)} j(z) \equiv \mathcal{E}_{2,7}(z) G_{7}(z) \quad\left(\bmod 7^{4}\right) \\
& \frac{E_{6}(z)}{E_{4}(z)} j(z) \equiv \mathcal{E}_{2,13}(z) G_{13}(z) \quad\left(\bmod 13^{2}\right) .
\end{aligned}
$$

We also need [4, Lemma 4].

Lemma 3.6 (Atkin [4]). [Atkin [4] We have

$$
\begin{aligned}
& j(z) \equiv G_{5}(z)+750+3^{2} \cdot 7 \cdot 5^{5} G_{5}(z)^{-1} \quad\left(\bmod 5^{8}\right), \\
& j(z) \equiv G_{7}(z)+748 \quad\left(\bmod 7^{4}\right), \\
& j(z) \equiv G_{13}(z)+70 \quad\left(\bmod 13^{2}\right) .
\end{aligned}
$$

Remark 3.7. In equation (3.50) we have corrected a misprint in [4, Lemma 4].

To handle the $(t, c)=(5,6)$ case of Theorem 1.6 we will need 


\section{Lemma 3.8.}

and

$$
\begin{aligned}
\frac{E_{6}(z)}{E_{4}(z)} j(z) & \equiv \mathcal{E}_{2,5}(z)\left(G_{5}(z)+2 \cdot 5^{5} G_{5}(z)^{-1}\right) \quad\left(\bmod 5^{6}\right) \\
\frac{E_{6}(z)}{E_{4}(z)} j(z)^{2} & \equiv \mathcal{E}_{2,5}(z)\left(2 \cdot 3 \cdot 5^{3} G_{5}(z)+G_{5}(z)^{2}\right) \quad\left(\bmod 5^{6}\right)
\end{aligned}
$$

$$
\frac{E_{6}(z)}{E_{4}(z)} j(z)^{a} \equiv \mathcal{E}_{2,5}(z)\left(\varepsilon_{1, a} G_{5}(z)^{a-2}+\varepsilon_{2, a} G_{5}(z)^{a-1}+G_{5}(z)^{a}\right) \quad\left(\bmod 5^{6}\right)
$$

for $a \geq 3$, where $\varepsilon_{1, a}, \varepsilon_{2, a}$ are integers satisfying

$$
\varepsilon_{1, a} \equiv 0 \quad\left(\bmod 5^{5}\right) \quad \text { and } \quad \varepsilon_{2, a} \equiv 0 \quad\left(\bmod 5^{3}\right) .
$$

Proof. The result can be proved from Lemmas 3.5 and 3.6, some calculation and an easy induction argument.

We need bases for $M_{2+12 s_{\ell}}\left(\Gamma_{0}(t)\right)$ for $t=5,7,13$. The following result follows from [7] and by checking the modular forms involved are holomorphic at the cusps $i \infty$ and 0 .

Lemma 3.9. Suppose $t=5,7$ or 13 and $\ell>3$ is prime. Then

$$
\operatorname{dim} M_{2+12 s_{\ell}}\left(\Gamma_{0}(t)\right)=1+(1+t) s_{\ell},
$$

and the set

$$
\left\{\mathcal{E}_{2, t}(z) \Delta(z)^{s_{\ell}} G_{t}(z)^{a}:-t s_{\ell} \leq a \leq s_{\ell}\right\}
$$

is a basis for $M_{2+12 s_{\ell}}\left(\Gamma_{0}(t)\right)$.

We are now ready to prove Theorem 1.6. We have two cases:

Case 1. In the first case we assume that $(t, c)=(5,5),(7,4)$ or $(13,2)$. Suppose $\ell>3$ is prime and $\ell \neq t$. By (2.24) we have

$$
\mathcal{A}_{\ell}(z)=\sum_{n=1}^{s_{\ell}} b_{n, \ell} \frac{E_{6}(z)}{E_{4}(z)} \frac{j(z)^{n}}{\eta(z)} .
$$

By Theorem 1.1, equation (2.21) and Lemma 3.9 we have

$$
\mathcal{A}_{\ell}(z)=\sum_{n=-t s_{\ell}}^{s_{\ell}} d_{n, \ell} \frac{\mathcal{E}_{2, t}(z)}{\eta(z)} G_{t}(z)^{n},
$$

for some integers $d_{n, \ell}\left(-t s_{\ell} \leq n \leq s_{\ell}\right)$. Now let

$$
K(z)=\sum_{n=1}^{s_{\ell}} d_{n, \ell} G_{t}(z)^{n} .
$$

By using Lemmas 3.5 and 3.6 to reduce equation (3.59) modulo $t^{c}$ and comparing the result with (3.60) we deduce that

$$
\mathcal{A}_{\ell}(z) \equiv \mathcal{E}_{2, t}(z) \frac{K(z)}{\eta(z)} \quad\left(\bmod t^{c}\right)
$$

and that

$$
d_{n, \ell} \equiv 0 \quad\left(\bmod t^{c}\right),
$$


for $-t s_{\ell} \leq n \leq 0$. By examining (3.60) we see that

$$
\mathcal{A}_{\ell}(z)=\mathcal{E}_{2, t}(z) \frac{K(z)}{\eta(z)}+O\left(q^{-\frac{1}{24}}\right)
$$

So if we let

$$
\mathcal{E}_{2, t}(z) \frac{K(z)}{\eta(z)}=\sum_{n=-s_{\ell}}^{\infty} \beta_{t, \ell}(n) q^{n-\frac{1}{24}},
$$

then (3.62) may be rewritten as

$$
\mathbf{a}\left(\ell^{2} n-s_{\ell}\right)+\chi_{12}(\ell)\left(\left(\frac{1-24 n}{\ell}\right)-1-\ell\right) \mathbf{a}(n)+\ell \mathbf{a}\left(\frac{n+s_{\ell}}{\ell^{2}}\right) \equiv \beta_{t, \ell}(n) \quad\left(\bmod t^{c}\right)
$$

and from (3.64) we have

$$
\mathbf{a}\left(\ell^{2} n-s_{\ell}\right)+\chi_{12}(\ell)\left(\left(\frac{1-24 n}{\ell}\right)-1-\ell\right) \mathbf{a}(n)+\ell \mathbf{a}\left(\frac{n+s_{\ell}}{\ell^{2}}\right)=\beta_{t, \ell}(n)
$$

for $-s_{\ell} \leq n \leq-1$. Equation (3.67) implies that

$$
\beta_{t, \ell}\left(-s_{\ell}\right)=-\ell
$$

and

$$
\beta_{t, \ell}(n)=0,
$$

for $-s_{\ell} \leq n \leq-1$. We can now apply Proposition 3.4 with $m=-s_{\ell}$ since $1-24 m=\ell^{2}$ and $t \neq \ell$. Hence

$$
\beta_{t, \ell}(n)=0, \quad \text { provided } \quad\left(\frac{1-24 n}{t}\right)=-1 .
$$

This gives Theorem 1.6 when $(t, c)=(5,5),(7,4)$ or $(13,2)$ by (3.66).

Case 2. We consider the remaining case $(t, c)=(5,6)$ and assume $\ell>5$ is prime. We proceed as in Case 1. This time when we use Lemma 3.8 to reduce (2.24) modulo $5^{6}$ we see that the only extra term occurs when $n=1$. We find that with $K(z)$ as before we have

$$
\mathcal{A}_{\ell}(z) \equiv \mathcal{E}_{2, t}(z) \frac{K(z)}{\eta(z)}+b_{1,5} \cdot 2 \cdot 5^{5} \frac{\mathcal{E}_{2,5}(z)}{\eta(z)} G_{5}(z)^{-1} \quad\left(\bmod 5^{6}\right) .
$$

All that remains is to show that

$$
b_{1,5} \equiv 0 \quad(\bmod 5),
$$

since then (3.62) actually holds modulo $5^{6}$ and the rest of the proof proceeds as in Case 1 . Since $E_{4}(z) \equiv 1(\bmod 5)$ we may reduce (2.18) modulo 5 to obtain

$$
\ell \Xi_{\ell} \equiv\left(\chi_{12}(\ell) \ell(1+\ell)-c_{0, \ell}\right) \frac{E_{6}(z)}{E_{4}(z)} \frac{1}{\eta(z)}-\sum_{n=1}^{s_{\ell}}(24 n+1) c_{n, \ell} \frac{E_{6}(z)}{E_{4}(z)} \frac{j(z)^{n}}{\eta(z)} \quad(\bmod 5) .
$$

But

$$
\mathcal{S}_{\ell}(z) \equiv 0 \quad(\bmod 5)
$$

by Theorem 1.3 (ii). Hence

$$
\ell \mathcal{A}_{\ell}(z) \equiv\left(\chi_{12}(\ell) \ell(1+\ell)-c_{0, \ell}\right) \frac{E_{6}(z)}{E_{4}(z)} \frac{1}{\eta(z)}-\sum_{n=1}^{s_{\ell}}(24 n+1) c_{n, \ell} \frac{E_{6}(z)}{E_{4}(z)} \frac{j(z)^{n}}{\eta(z)} \quad(\bmod 5)
$$


and we see that $b_{1,5}$ the coefficient of $\frac{E_{6}(z)}{E_{4}(z)} \frac{j(z)}{\eta(z)}$ is divisible by 5 as required. This completes the proof of Theorem 1.6.

We close by illustrating Theorem 1.6 when $t=5$ and $\ell=7$. In this case the theorem predicts that

$$
\mathbf{a}(49 n-2)-\left(\frac{1-24 n}{7}\right) \mathbf{a}(n)+7 \mathbf{a}\left(\frac{n+2}{49}\right) \equiv-8 \mathbf{a}(n) \quad\left(\bmod 5^{6}\right),
$$

when $n \equiv 1,2(\bmod 5)$. When $n=1$ this says

$$
149077845 \equiv-280 \quad\left(\bmod 5^{6}\right),
$$

which is easy to check.

\section{Acknowledgements}

I would like to thank Ken Ono for sending me preprints of his recent work [12, [13].

\section{REFERENCES}

1. G. E. Andrews, The number of smallest parts in the partitions of n, J. Reine Angew. Math. 624 (2008), 133-142. URL:http://dx.doi.org/10.1515/CRELLE.2008.083

2. A. O. L. Atkin and F. G. Garvan, Relations between the ranks and cranks of partitions, Ramanujan J. 7 (2003), 343-366.

URL:http://dx.doi.org/10.1023/A:1026219901284

3. A. O. L. Atkin, Ramanujan congruences for $p_{-k}(n)$, Canad. J. Math. 20 (1968), 67-78; corrigendum, ibid. 21 (1968), 256. URL:http://cms.math.ca/cjm/v20/p67

4. A. O. L. Atkin, Multiplicative congruence properties and density problems for $p(n)$, Proc. London Math. Soc. (3) 18 (1968), 563-576. URL:http://dx.doi.org/10.1112/plms/s3-18.3.563

5. B. C. Berndt and R. J. Evans, The determination of Gauss sums, Bull. Amer. Math. Soc. (N.S.) 5 (1981), 107129.

URL:http://dx.doi.org/10.1090/S0273-0979-1981-14930-2

6. K. Bringmann, On the explicit construction of higher deformations of partition statistics, Duke Math. J. 144 (2008), 195-233.

URL: http://dx.doi.org/10.1215/00127094-2008-035

7. H. Cohen and J. Oesterlé, Dimensions des espaces de formes modulaires, Springer Lecture Notes, Vol. 627, 1977, $69-78$.

URL:http://dx.doi.org/10.1007/BFb0065297

8. A. Folsom and K. Ono, The spt-function of Andrews, Proc. Natl. Acad. Sci. USA 105 (2008), 20152-20156. URL:http://mathcs.emory.edu/ ono/publications-cv/pdfs/111.pdf

9. K. C. Garrett, Private communication, October 18, 2007.

10. F. G. Garvan, Congruences for Andrews' smallest parts partition function and new congruences for Dyson's rank, Int. J. Number Theory 6 (2010), 1-29. URL: http://dx.doi.org/10.1142/S179304211000296X

11. F. G. Garvan, Congruences for Andrews' spt-function modulo powers of 5, 7 and 13, in preparation. URL:http://www . math.ufl.edu/ fgarvan/papers/spt2.pdf

12. K. Ono, Congruences for the Andrews spt-function, Proc. Natl. Acad. Sci. USA, to appear. URL: http://mathcs. emory.edu/ ono/publications-cv/pdfs/132.pdf

13. K. Ono, The partition function and Hecke operators, preprint.

14. J.-P. Serre, Formes modulaires et fonctions zêta p-adiques in "Modular functions of one variable, III", (Proc. Internat. Summer School, Univ. Antwerp, 1972), pp. 191-268, Lecture Notes in Math., Vol. 350, Springer, Berlin, 1973.

URL: http://dx.doi.org/10.1007/978-3-540-37802-0_4

Department of Mathematics, University of Florida, Gainesville, Florida 32611-8105

E-mail address: fgarvan@ufl.edu 